\title{
Nível de satisfação e dor de mulheres que realizaram epilação com laser de diodo.
}

\section{Level of satisfaction and pain of women who underwent hair removal laser diode}

Camila Rabêlo Barbosa1; Rodrigo Disconzi Nunes²; Sávia Denise Silva Carlotto Herrera3; Edison Benedito da Luz Brito Junior ${ }^{4}$; Tayla Santos Querido Disconzi ${ }^{5}$; Thaynara Ludvig6
RESUMO

Introdução: O laser de diodo é uma técnica de fácil acesso e está entre uma das mais utilizadas para fins epilatórios, sendo que nos últimos anos tem surgido como uma opção de alta eficácia, longa duração e efeitos colaterais reduzidos. Objetivo: Avaliar o nível de satisfação e dor de pacientes que realizaram epilação com laser de diodo. Material e Método: Trata-se de uma pesquisa quantitativa e descritiva, composta por mulheres que foram submetidas ao procedimento de epilação a laser de diodo, o LightSheer ET®.no período de março e abril de 2015, Que responderam dois questionários, um relacionado ao nível de satisfação e outro referente a dor, os resultados obtidos foram organizados em planilha do programa Excel®,realizadas frequências para todas as variáveis e análise de média e mediana para a idade. Resultados: Foram incluídas 19 pacientes com idade entre 18 e 43 anos (mediana 33 e média 32), com prevalência de fototipo de Fitzpatrick III $(52,63 \%)$. Em relação a satisfação com o resultado, $8(42,10 \%)$ relataram nível " excelente" com a satisfação. Em relação a dor referida através da Escala Visual Analógica (EVA), 3 pacientes $(15,79 \%)$ relataram o grau de dor máximo. Conclusão: Com a realização do presente estudo observou-se que, existe um alto nível de satisfação quanto a epilação a laser, conhecida como definitiva e a dor é um fator importante, mas não limitante para o procedimento.

Palavras-chave: Epilação. Laser. Satisfação e dor.

\section{ABSTRACT}

Introduction: The diode laser is an easy technique and is among one of the most used for epilatórios purposes, and in recent years has emerged as a highly effective option, long life and reduced side effects. Objective: To evaluate the level of satisfaction and pain of patients undergoing hair removal laser diode. Methods: This is a quantitative and descriptive research, consisting of women who were subjected to hair removal procedure diode laser, with the intuited removal of unwanted hair, occurring data collection between March and April 2015 inclusion criteria were: signing TCLE, being female and being treated for laser hair removal in Clinical and periods. Answered two questionnaires, one related to the level of satisfaction and other related pain, the results were organized into Excelß spreadsheet program. Frequencies were performed for all variables and mean and median analysis for age. Results: We included 19 patients aged 18 to 43 years (median 33 and 32 average), with a prevalence of skin type Fitzpatrick III (52.63\%). Regarding satisfaction with the result, 8 (42.10\%) reported level "excellent" with satisfaction. Regarding the referred pain by visual analog scale (VAS), 3 patients $(15.79 \%)$ reported the maximum degree of pain. Conclusion: With the completion of this study it was observed that there is a high level of satisfaction as the ultimate hair removal and pain is an important factor, but not limiting to the procedure.
${ }^{1}$ Fisioterapeuta Graduada pela Universidade de Gurupi (UnirG). Gurupi-TO, Brasil. $\quad$ Email: camilarabelo1@hotmail.co $\mathrm{m}$

${ }^{2}$ Fisioterapeuta. Mestre em Ciências da Saúde pela Universidade Federal de Goiás- Goiania (GO). Professor da Universidade de Gurupi (UnirG). GurupiTO, Brasil.

${ }^{3}$ Fisioterapeuta. Mestra em Ciências da Saúde pela Universidade Federal do Tocantins- Palmas (TO). Professora da Universidade de Gurupi (UnirG). GurupiTO, Brasil.

${ }^{4}$ Acadêmico do Curso de Medicina da Universidade de Gurupi (UnirG). LimeiraSP, Brasil.

${ }^{5}$ Fisioterapeuta. Graduada pela Universidade de Gurupi (UnirG). Especialista em Dermatofuncional (IEES). Gurupi-TO, Brasil.

${ }^{6}$ Médica. Graduada pela Universidade de Gurupi (UnirG). Especialista em Medicina da Familia e Comunidade. Preceptora da Residência em Medicina da Família e Comunidade.

Keywords: Epilation. Diode. Satisfaction and pain. 


\section{INTRODUÇAO}

Atualmente há diversas formas de remoção dos pelos corporais, pois As pessoas que sofrem com excesso de pelos, e também aquelas que querem a sua redução por comodidade ou estética estão cada dia mais procurando métodos efetivos para sua remoção. ${ }^{24-25}$.Os pelos indesejados do corpo passaram a ser um problema estético muito comum para mulheres e homens. Para sua remoção são utilizadas técnicas manuais, como os métodos físicos (ceras ou barbeadores) e os métodos químicos (ácidos tioglicolato de cálcio ou tioglicolato de potássio), e técnicas que utilizam procedimentos clínicos, chamados de depilação ou epilação definitiva (laser, eletrólise, luz intensa pulsada) ${ }^{1}$, que correspondem ao ato de arrancar os pelos ou resultado da remoção deles. ${ }^{2-3}$

Uma das técnicas utilizadas para epilação é o Laser (Light Amplification by the Stimulated Emission of Radiation). A intensidade e duração do pulso fazem com que os feixes de luz, com cores particulares produzidas por ele, se diferenciem de ultravioleta até a luz infravermelha, dependendo do objetivo para o qual será empregado. ${ }^{4}$

Os comprimentos de onda mais longos penetram mais profundamente na pele, assim no intervalo de $600-1100 \mathrm{~nm}$ a absorção pela melanina pode ser usada para fototermólise seletiva, que é o mecanismo de ação utilizado pelo laser. A lesão térmica é provocada por pulsos de radiação, que são absorvidos de maneira seletiva pelo cromóforoalvo, a melanina, que corresponde ao principal cromofóro existentes nos folículos pilosos e a energia produzida pelo laser é atraída pela melanina, com esse aquecimento ocorre 0 enfraquecimento ou destruição do folículo piloso. .-6-7-8 $^{-6}$

Os tipos de laser mais eficazes para a epilação são: Laser de rubi $(695 \mathrm{~nm})$, de alexandrite $(755 \mathrm{~nm})$, de diodo $(800 \mathrm{~nm})$ e o Nd: YAG $(1.064 \mathrm{~nm})$. As taxas médias de redução de pelos, com essa técnica, são relatados entre $70 \%$ e 90\% por no mínimo de 6 meses de tratamento. Esse tempo de tratamento está ligado ao ciclo de crescimento natural do pelo, onde durante 0 tratamento estarão em várias fases, sendo elas: anágena (crescimento), catágena (estabilidade) e tológena (queda). A ação do laser é melhor na fase anágena, os pelos que não estiverem nesta fase não terão uma eficácia no tratamento, porque só fios anágenos são particularmente sensíveis às agressões físicas, devido ao fato da melanina do pelo somente ser encontrada no bulbo piloso quando este se encontra nesta fase, por isso a necessidade de sessões regulares por um certo período de tempo. ${ }^{9-10-11-}$ 
O Laser de Diodo é considerado o mais indicado para a técnica de epilação, este tipo de laser possui um comprimento de onda que varia de $800-810 \mathrm{~nm}$, esse parâmetro é confiável para não lesionar a epiderme e é bem absorvido pela melanina, ${ }^{16-17-18}$ onde ocorre um superaquecimento do folículo, dissipando o calor para as estruturas ao redor, como a veia e artéria que nutri o folículo e locais próximos ao músculo eretor do pelo, fazendo com que o procedimento seja ainda mais eficaz, um tipo específico deste laser é o LightSheer $\mathrm{ET} \circledast{ }^{19}$

Este tipo de tratamento tem maior procura por mulheres com a idade entre 16 e 50 anos $^{1,20-21}$, apresentando um elevado nível de satisfação com os resultados obtidos, onde em estudo prévio, observou que 155 (90\%) pacientes ficaram satisfeitos com o tratamento ${ }^{1,17}$. Sendo que o nível de dor durante o procedimento com o laser de diodo é bem variável, pois a sensibilidade ao estímulo doloroso é diferente em cada indivíduo. ${ }^{6,9}$

Assim, este estudo teve por finalidade avaliar o nível de satisfação e grau de dor das pacientes que se submeteram a epilação com laser de diodo.

\section{MATERIAIS E METODOS}

O presente estudo foi submetido ao Comitê de Ética e Pesquisa da Universidade de Gurupi (UNIRG) e foi aprovado sob o número de protocolo 1.023.106, seguindo as conformidades da resolução 466/12, referente a pesquisas envolvendo seres humanos. Trata-se de uma pesquisa quantitativa e descritiva, onde foram incluídas 19 mulheres com idade entre 18 e 43 anos submetidas ao procedimento de epilação a laser de diodo com o intuído de remoção de pelos indesejáveis.

A coleta de dados foi realizada no período de março e abril de 2015, onde foram avaliados o nível de satisfação e dor de pacientes atendidas na Clínica Carla von Glehn Dermatologia \& Saúde, localizada na Av. Piauí, 1640 - S Central, Gurupi - TO, 77410-030.

Como critérios de inclusão foram adotados os seguintes parâmetros: assinar o termo de consentimento livre esclarecido (TCLE), ser do sexo feminino e estar em tratamento para epilação.Como critérios de exclusão foram adotados os seguintes parâmetros: ser do sexo masculino, não assinar o TCLE, faltar a sessão no dia da entrevista e estar em tratamento na clínica fora do período de análise, março e abril de 2015.

O laser de diodo, utilizado na presente pesquisa, é fabricado pela empresa Lumenis, possui um comprimento de onda de $800 \mathrm{~nm}$, fluência de $10-60 \mathrm{~J} / \mathrm{cm} 2$, com 
duração de pulso que varia de $5 \mathrm{~ms}$ a $100 \mathrm{~ms}$. Possui uma ponteira de safira (ChillTip ${ }^{\mathrm{TM}}$ ), que toca diretamente a pele do paciente e serve para um resfriamento da epiderme. Sendo o mesmo indicado para todos os tipos de pele, baseado no foto tipo Fitzpatrick, ${ }^{22}$ onde inclui também pele bronzeada. A aplicação do laser foi executada por uma profissional devidamente treinada, utilizando o Laser de Diodo LightSheer ET®.

Durante a sessão esta profissional observa a classificação do fototipo de Fitzpatrick sendo: I - Branca, sempre queima, nunca bronzeia e muito sensível ao Sol, II - Branca, sempre queima, Bronzeia muito pouco e sensível ao Sol, III - Morena clara, queima (moderadamente), Bronzeia (moderadamente) e sensibilidade normal ao Sol, IV - Morena moderada, queima (pouco), sempre bronzeia e uma sensibilidade normal ao Sol, V Morena escura, queima (raramente), sempre bronzeia e sendo pouco sensível ao Sol, VI Negra, nunca queima, totalmente pigmentada e totalmente insensível ao Sol.

Após a sessão, quando as pacientes foram liberadas, ocorreu a abordagem pela pesquisadora. As pacientes foram convidadas a participar da pesquisa sendo esclarecido o motivo e como seria realizada a mesma.

Posteriormente, após aceitarem e assinarem o TCLE, responderam os questionários previamente elaborados, contendo 13 perguntas relacionadas ao nível de satisfação como: Em relação ao local tratado: qual a sua satisfação com o resultado?; Em relação ao tempo para aparecer os resultados satisfatórios; O valor empregado no tratamento condiz com a satisfação dos resultados?; onde as mesmas foram respondidas através de uma qualificação contendo 5 alternativas (Péssimo, Ruim, Bom, Ótimo, Excelente) ou avaliadas através das respostas sim ou não, e outras 6 referentes a dor, onde o questionário foi composto por uma Escala Visual Analógica (EVA), que consiste em auxiliar na graduação da intensidade da dor referida, uma tabela avaliação dividida em três grupos (sensitiva, afetiva e avaliativa) e questões como: Quanto tempo após a sessão você ainda continua sentindo a dor?; A dor, relacionada, poderia fazer você desistir do tratamento?

Os questionários foram aplicados no período de março e abril de 2015 e os dados coletados foram empregados exclusivamente para os propósitos da pesquisa realizada, sendo que as informações confidenciais como nome, telefone, enderenço entre outras não foram divulgadas. 
DOI 10.18606/2318-1419/amazonia.sci.health.v7n3p111-121
Nível de satisfação e dor de mulheres que realizaram epilação com laser de diodo.

Barbosa, CR. Nunes, RD. Herrera, SDSC. Júnior, EBLB. Disconzi, TSQ. Ludvig, T.

Os resultados obtidos foram organizados em planilha do programa Excel@. Foram realizadas frequências para todas as variáveis e análise de média e mediana para a idade.

\section{RESULTADOS}

Durante o estudo foram incluídas 19 pacientes com idade entre 18 e 43 anos (mediana 33 e média 32), com prevalência de fototipo Fitzpatrick III (52,63\%). Entre elas, 11 pacientes $(57,89 \%)$ tinham ensino superior completo e $8(42,10 \%)$ com uma renda de 3 a 9 salários mínimos.

Em relação a quantidade de áreas tratadas, 14 (73,69\%) estavam tratando apenas uma área, sendo que $5(26,31 \%)$ relataram mais de uma área tratada na mesma sessão. A área mais tratada por elas foi respectivamente: virilha $(37,5 \%)$, axila $(29,17 \%)$ e buço $(20,87 \%)$. Sendo que, perna, face e pescoço apresentaram apenas uma paciente em tratamento $(4,16 \%)$.

Já em relação a satisfação com o resultado, 6 pacientes $(31,58 \%)$ estavam na primeira sessão, onde, não foi possível identificar se estavam satisfeitas. Das pacientes que se pode coletar este dado, 8 (42,10\%) relataram nível " excelente" com a satisfação.

O nível de satisfação na sessão, referido por 7 pacientes, também foi " excelente" $(36,84 \%)$. Logo, observou-se o mesmo em relação ao tempo para aparecer os resultados $(36,84 \%)$.

Das pacientes participantes do estudo, $13(68,42 \%)$ relataram ainda não ter realizado epilação em outras áreas, entre tanto das 6 (31,58\%) que realizaram, 2 (33,33\%) referiram ter feito em 2 locais a mais e 4 (66,67\%) em apenas um. Destes locais 1 (12,5\%) realizou na axila onde foi referido um "ótimo" resultado e nos demais, observou-se um resultado " excelente", apresentando de modo respectivo: Axila (62,5\%), virilha (12,5\%) e buço (12,5\%). Já para saber se o nível de satisfação variou de um local tratado para outro, 4 pacientes $(66,67 \%)$ relataram que sim.

Das 19 Pacientes participantes do estudo, 18 (94.74\%) relataram que o valor empregado nas sessões condiz com a satisfação esperada pelo tratamento (Tabela 1). 
DOI 10.18606/2318-1419/amazonia.sci.health.v7n3p111-121 Revista Amazônia Science \& Health ANO Volume/Número
Nível de satisfação e dor de mulheres que realizaram epilação com laser de diodo.

Barbosa, CR. Nunes, RD. Herrera, SDSC. Júnior, EBLB. Disconzi, TSQ. Ludvig, T.

Tabela 1 - Nível de satisfação das pacientes incluídas no estudo (n=19)

\begin{tabular}{lll} 
Variável & $\mathbf{N}$ & $\%$ \\
\hline Foto tipo & & \\
I & - & - \\
II & 7 & 36,84 \\
III & 10 & 52,63 \\
IV & 2 & 10,53 \\
V & - & - \\
VI & - & -
\end{tabular}

Idade

16-20

21-30

$31-40$

$41-50$

Escolaridade

Ensino fundamental incompleto

Ensino fundamental completo

Ensino médio incompleto

Ensino médio completo

Ensino superior incompleto

Ensino superior completo

Renda (salário mínimo)

Não Tem

Até 1

$1-3$

3-9

Acima de 9

Quantidade de áreas tratadas

1

2

3

Satisfação com o resultado

Excelente

Ótimo

Bom

Ruim

Péssimo

Não possível de avaliação*

\section{Sessão}

1

2

3

4

5

6

$>6$

Nível de satisfação na sessão

Excelente

Ótimo
1

5,26

6

10

31,58

2

52,64

15,79

5

11

36,84

10,53

10,52

26,32

57,89

26,32

26,32

42,10

5,26

14

73,69

21,05

4

5,26

42,10

15,79

5,26

5,26

31,58

6

31,58

36,84

10,53

5,26

10,53

$1 \quad 5,26$

$7 \quad 36,84$

$3 \quad 15,79$ 
DOI 10.18606/2318-1419/amazonia.sci.health.v7n3p111-121
Nível de satisfação e dor de mulheres que realizaram epilação com laser de diodo.

Barbosa, CR. Nunes, RD. Herrera, SDSC. Júnior, EBLB. Disconzi, TSQ. Ludvig, T.

Bom

Ruim

Péssimo

Não possível de avaliação*

Tempo para aparecer o resultado

Excelente

Ótimo

Bom

Ruim

Péssimo

Não possível de avaliação*

Epilação em mais de uma área

Não

Sim

Nível de satisfação variou de um lugar para

outro

Não

Sim

Valor empregado condiz com a satisfação

Não

Sim

* pacientes na primeira sessão, número (n), porcentagem (\%)

Em relação a dor referida através da Escala Visual Analógica (EVA), 3 pacientes $(15,79 \%)$ relataram o grau de dor máximo. Já em relação a classificação sensitiva, 16 pacientes $(84,2 \%)$ referiram mais de uma sensação dolorosa, onde $12(28,57 \%)$, das 19 pacientes, declararam a dor em queimação a mais sentida. Na classificação afetiva, 12 $(63,16 \%)$ referiram apenas uma sensação, onde $5(26,31 \%)$ referiram a sensação cansada a mais citada. Na classificação avaliativa, 17 (89,47\%) disseram que a dor apenas incomodava.

Sobre tempo de dor após a sessão 11 pacientes $(57,89 \%)$ referiram que a sensação de dor acabava antes das 24 horas e que a dor com o passar das sessões melhorou em 8 $(42,10 \%)$

Foi observado que das $8(42,11 \%)$ pacientes que referiram ter feito epilação em outras áreas, 7 (87,5\%) delas relataram a virilha como a mais dolorida, sendo que 2 pacientes realizaram a epilação em mais de uma área ao mesmo tempo.

Quanto a possível desistência do tratamento, 18 (94,78\%) informaram que não desistiriam da epilação por causa da dor (Tabela 2). 
DOI 10.18606/2318-1419/amazonia.sci.health.v7n3p111-121 Revista Amazônia Science \& Health ANO Volume/Número
Nível de satisfação e dor de mulheres que realizaram epilação com laser de diodo.

Barbosa, CR. Nunes, RD. Herrera, SDSC. Júnior, EBLB. Disconzi, TSQ. Ludvig, T.

Tabela 2- Nível de dor das pacientes incluídas no estudo ( $n=19)$

\begin{tabular}{l}
\hline Variável \\
\hline Grau de dor \\
1 \\
2 \\
3 \\
4 \\
5 \\
6 \\
7 \\
8 \\
9 \\
10
\end{tabular}

\begin{tabular}{ll}
$\mathbf{N}$ & $\%$ \\
\hline 1 & 5,26 \\
- & - \\
4 & 21,05 \\
1 & 5,26 \\
2 & 10,53 \\
2 & 10,53 \\
2 & 10,53 \\
3 & 15,79 \\
1 & 5,26 \\
3 & 15,79
\end{tabular}

Classificação da dor (sensitiva)*

Latejante

Pontada

Choque

Fina-agulha

Fisgada

Queimação

16,67

21,43

Dolorida

9,52

28,57

Classificação da dor (afetiva)*

Cansativa

Enjoada

Sufocante

Apavorante

10,53

Aborrecida

10,53

15,79

Nenhuma

36,84

Classificação da dor (avaliativa)

Que incomoda

Insuportável

Tempo de dor após a sessão

Não possível de avaliação **

Até 24 horas

Após 24 horas

A dor com o passar das sessões melhora

Não possível de avaliação **

Não

31,58

Sim

6

Tratamento em mais de um local

Não

11

57,89

Sim

Tratamento em mais de um local qual a maior dor

Axila

Virilha

Buço

Perna

Braço 


\section{Outros}

Desistir por causa da dor
Não
Sim
*Paciente poderia relatar mais de uma sensação, **pacientes na primeira sessão, número (n),
ntagem (\%).

\section{DISCUSSAO}

O laser de diodo Light Sheer® (Lumenis), está entre uma das alternativas mais usadas para epilação, neste estudo as 19 mulheres participantes foram submetidas a este tipo de laser, onde procurou saber o nível de satisfação e dor das mesmas. Destas, o estudo demonstrou a prevalência de idade entre 18 e 43 anos, corroborando com o estudo de Souza $^{1}$, onde foi relatado que participaram do estudo mulheres com idades entre 18 e 45 anos, comparando com o mesmo estudo, foi observado a prevalência do fototipo fitzpatrick III, onde apresentou 5 pacientes com fototipo II e 10 com fototipo III e neste estudo a prevalência de fototipo Fitzpatrick III com 10 pacientes (52,63\%).

Em relação a satisfação com o resultado o presente estudo demonstrou que 8 pacientes $(42,10 \%)$ apresentaram um resultado "excelente" e onde apenas uma $(5,26 \%)$ relatou estar insatisfeita, ao olhar para as causas da sua insatisfação foi devido ao pelo continuar se mantendo, confirmando os achados de Adamič ${ }^{17}$, onde de 173 pacientes, 150 (90\%) pacientes estavam satisfeitas com o tratamento (40\% muito satisfeitas e $50 \%$ satisfeitas).

De acordo com o tempo para aparecer os resultados, na pesquisa de Naves ${ }^{16} 0$ tempo médio foi de 3 meses, sendo que, o intervalo entre as sessões foram de 2 a 3 meses, onde os mesmos relataram que este intervalo foi referente as fases de crescimento do pelo, devido o tratamento só ter eficácia caso o pelo esteja na fase anágena, corroborando com este estudo, onde 7 pacientes (36,84\%) apresentaram um nível de satisfação " excelente" em relação ao tempo para obter resultados.

Sobre a realização da epilação em outras áreas, 6 pacientes $(31,58 \%)$ referiramque realizaram, apresentando porcentagem parecida com o de Ibrahimi ${ }^{23}$, onde $17 \%$ de 34 pacientes haviam realizado epilação a laser anteriormente em outras áreas. 
DOI 10.18606/2318-1419/amazonia.sci.health.v7n3p111-121 Revista Amazônia Science \& Health ANO Volume/Número
Nível de satisfação e dor de mulheres que realizaram epilação com laser de diodo.

Barbosa, CR. Nunes, RD. Herrera, SDSC. Júnior, EBLB. Disconzi, TSQ. Ludvig, T.

O estudo de lbrahimi ${ }^{23}$ relatou que a dor observada através da escala visual analógica foi referida pela maioria das mulheres como moderada $(21,05 \%)$, confirmando nossos achados, onde apenas 3 pacientes (15,79\%) relataram o grau de dor máximo.

Em relação ao tempo de dor após a sessão 11 pacientes $(57,89 \%)$ referiram que a sensação de dor acabava antes das 24 horas, o que condiz com os achados de Rao, et al ${ }^{7}$, onde ele relata que as pacientes submetidas a sessão de laser referiram melhora quase que imediatamente após cada sessão.

Já a dor com o passar das sessões melhorou em 8 pacientes (42,10\%), não condizendo com estudo de Ibrahimi ${ }^{23}$, onde os níveis de dor foram ligeiramente piores no terceiro tratamento do que no primeiro tratamento, isto pode ser relacionado ao aumento das fluências com o passar das sessões no estudo de lbrahimi, et al ${ }^{23}$.

\section{CONSIDERAÇOES FINAIS}

Conclui-se que, existe um alto nível de satisfação quanto a epilação definitiva com uso de Laser de diodo, e a dor é um fator importante, mas não limitante para o procedimento.

\section{REFERÉNCIAS}

1 - Souza FHM, Ribeiro CF, Weigert S, Schmidt JV, Fabricio LZ, Ataíde DST. Estudo comparativo de uso de Laser de diodo (810nm) versus luz intensa pulsada (filtro 695nm) em epilação axilar. Surg Cosmet Dermatol, Curitiba, jun-set, 2010;2(3):185-90.

2- Rey L. Dicionário de termos técnicos de Medicina e Saúde. Rio de Janeiro: Guanabara; 2003.

3- Stedman TL. Dicionário Médico. Rio de Janeiro: Guanabara; 2003.

4- Borges FS. Modalidades terapêuticas nas disfunções Estéticas. 2. ed. SãoPaulo: Phorte, 2006

5- Sociedade Brasileira de Dermatologia. Portal da Sociedade Brasileira de Dermatologia Laser [acesso em 16 de nov 2014]. Disponivel em: http://www.sbd.org.br/tratamentos/laser/.

6- Mandt N, Troilius A, Drosnerz, M. Epilation today: physiology of the hair follicle and clinical photo-epilation. J Investig Dermatol Symp Proc, Berlin, dez, 2005;10(3):271-4.

7- Rao J, Goldman MP. Prospective, Comparative Evaluation of Three Laser Systems Used Individually and in Combination for Axillary Hair Removal. Dermatol Surg, dez, 2005;31(12):1671-7.

8- Drummond AMC. Confiabilidade metrológica de equipamentos eletromédicos a laser e a luz intensa pulsada. Dissertação (Mestrado) - Programa de Pós-graduação de Metrologia para Qualidade e Inovação, Pontifícia Universidade Católica do Rio de Janeiro, Rio de Janeiro, 2007:120.

9- Kede M, Sabatovich O. Dermatologia Estética. São Paulo: Atheneu, 2004. 
DOI 10.18606/2318-1419/amazonia.sci.health.v7n3p111-121 Revista Amazônia Science \& Health ANO Volume/Número
Nível de satisfação e dor de mulheres que realizaram epilação com laser de diodo.

Barbosa, CR. Nunes, RD. Herrera, SDSC. Júnior, EBLB. Disconzi, TSQ. Ludvig, T.

10- Pereira JM. Propedêutica das doenças dos cabelos. São Paulo: Atheneu, 2001

11- Goldberg DJ, Samady JA. Evaluation of a long-pulse Qswitched Nd: YAG laser for hair removal. Dermatol Surg, fev, 2000;26(2):109-13.

12- Patriota, RCR. Laser um aliado na dermatologia. Rev Med. São Paulo, abr-jun,2007;86(2):64-70.

13- Catorze, MG. Laser: fundamentos e indicações em dermatologia. Med Cutan Iber Lat Am. Lisboa, 2009;37(1):5-27.

14- Grossman MC, Dierickx C, Farinelli W, Flotte T, Anderson RR. Damage to hair follicles by normal mode ruby laser pulses. J Am Acad Dermatol, dez,1996;35(6):889-94.

15- Liew SH, Cerio R, Sarathchandra P, Grobbelaar AO, Gault DT, Sanders R, Green C. et al. Ruby assisted hair removal: An ultrastructural evaluation of cutaneous damage. Br J Plast Surg, dez,1999;52(8):636-43.

16- Naves APC, Assunção FFO. Epilação Progressiva usando Laser Diodo e Luz intensa pulsada: Uma revisão da Literatura atual. Revista Inspirar Movimento \& Saúde, mar./abr,2010;2(2):35-8.

17- Adamič M, Pavlović MD, Stamenković M, Nenadić D. Hair removal in women with an 800-nm diode laser: self-reported satisfaction and expectations from treatment are not the same. Serb J Dermatol Venereology, 2009; 4:141-6.

18- Lou WW, Quintana AT, Geronemus RG, Grossman MC. Prospective study of hair reduction by diode laser (800nm) with long-term follow-up. Dermatol Surg, mai, 2000 ;26(5):428-32.

19- Cameron $\mathrm{H}^{1}$, Ibbotson SH, Dawe RS, Ferguson J, Moseley $\mathrm{H}$. Within-patient right-left blinded omparison of diode $(810 \mathrm{~nm})$ laser therapy and intense pulsed light therapy for hair removal. Laser Med Sci, out, 2008;23(4):393-7.

20- DeMaria AL, Flores M, Hirth JM, Berenson AB. Complications related to pubic hair removal. Am J Obstet Gynecol. Galveston,Texas, jun, 2014;210(6):528.e1-5.

21- Davoudi SM, Behnia F, Gorouhi F, Keshavarz S, Kashani MN, Firoozabadi MR. et al. Comparison of longpulsed alexandrite and Nd:YAG lasers, individually and in combination, for leg hair reduction: an assessorblinded, randomized trial with 18 months of follow-up. Arch Dermatol, Out, 2008;144(10):1323-7.

22- Battle EF, Anderson RR. Study of very long-pulsed (100ms) high-powered diode laser for hair reduction on all skin types. Coherent Medical, Santa Clara, CA, 2000.

23- Ibrahimi OA, Kilmer SL. Long-term clinical evaluation of a 800-nm long-pulsed diode laser with a large spot size and vacuum-assisted suction for hair removal. Dermatol Surg, jun, 2012;38(6):912-7.

24- Chi A, Schleder CJ, Lechiw T. Análise da efetividade do laser de diodo para depilação permanente em região de axila e virilha. Fisioterapia Brasil, v.16, n. 3, 2016.

25- Gonçalves CK, Simões NDP. Análise do Tratamento Epilatório Utilizando Laser de Diodo de $800 \mathrm{~nm}$ no Período de 2006-2011 na Cidade de Quedas do Iguaçu, PR. Rev Bras Terap e Saúde, 5(1):1-8, 2014. 